\title{
Metastatic renal cell carcinoma to the left maxillary sinus
}

\author{
Y.-F. He' ${ }^{1}$,J. Chen ${ }^{1}$, W.-Q. Xu' ${ }^{2}$, C.-S. Ji' ${ }^{1}$, J.-P. Du ${ }^{1}$, H.-Q. Luo ${ }^{1}$ and B. Hu${ }^{1}$ \\ ${ }^{1}$ Department of Medical Oncology, \\ The Provincial Hospital Affiliated to Anhui Medical University, Hefei, China \\ ${ }^{2}$ Department of Otorhinolaryngology, \\ The Provincial Hospital Affiliated to Anhui Medical University, Hefei, China
}

Corresponding author: B. Hu

E-mail:hubing3756@yeah.net

Genet. Mol. Res. 13 (3): 7465-7469 (2014)

Received July 23, 2013

Accepted January 13, 2014

Published September 12, 2014

DOI http://dx.doi.org/10.4238/2014.September.12.12

\begin{abstract}
Metastatic tumors in the paranasal sinuses are very rare. The origin of metastatic tumors in the paranasal sinuses is often renal cancer. Renal cell carcinomas are known for their tendency for early metastasis, and symptoms due to the metastatic lesion may be the only initial manifestation. In this paper, we deal with the case of a 35-year-old male patient who presented with a mass in the left maxillary region. The presence of a primary renal cell carcinoma was recognized only after surgical removal of the metastatic tumor. The presentation, diagnosis and treatment of this tumor are discussed with a review of the literature.
\end{abstract}

Key words: Renal cell carcinoma; Metastasis; Maxillary sinus 


\section{INTRODUCTION}

Primary carcinoma of the paranasal sinuses constitutes approximately $0.3 \%$ of all human cancers (Miyamoto and Helmus, 1973). Most malignant tumors occurring in the nasal and paranasal sinuses are primary tumors, and metastatic tumors in these areas are extremely rare (Hefer et al., 1994). Metastatic renal cell carcinoma to the paranasal sinus is seen as a mass in the left maxillary region. This symptom may precede that of the primary tumor.

\section{CASE REPORT}

A 35-year-old man was referred to the otolaryngology clinic with a mass in the left maxillary region. Additional review of symptoms revealed no other complaints, specifically no abdominal or urinary signs or symptoms. Past medical, family, and social history revealed no significant additional history.

Computed tomography (CT) scans of the paranasal sinus detected a mass in the left maxillary sinus. This mass was strongly enhanced on contrast-enhanced viewing. There was displacement of adjacent bone, and lytic bone lesions could be observed (Figure 1). The tumor was removed en bloc. The margins of resection were free of tumor. The pathologic findings were consistent with metastatic renal cell carcinoma (Figure 2). Immunohistochemical staining for cytokeratin, epithelial membrane antigen, and vimentin was positive and thus compatible with metastatic renal cell carcinoma. Immunohistochemical examination showed negative staining for S100 and melanoma-specific marker HMB-45. The metastatic foci contained focal, diastase-sensitive and periodic acid Schiff positive material, which was indicative of glycogen in the cytoplasm of the clear cells.

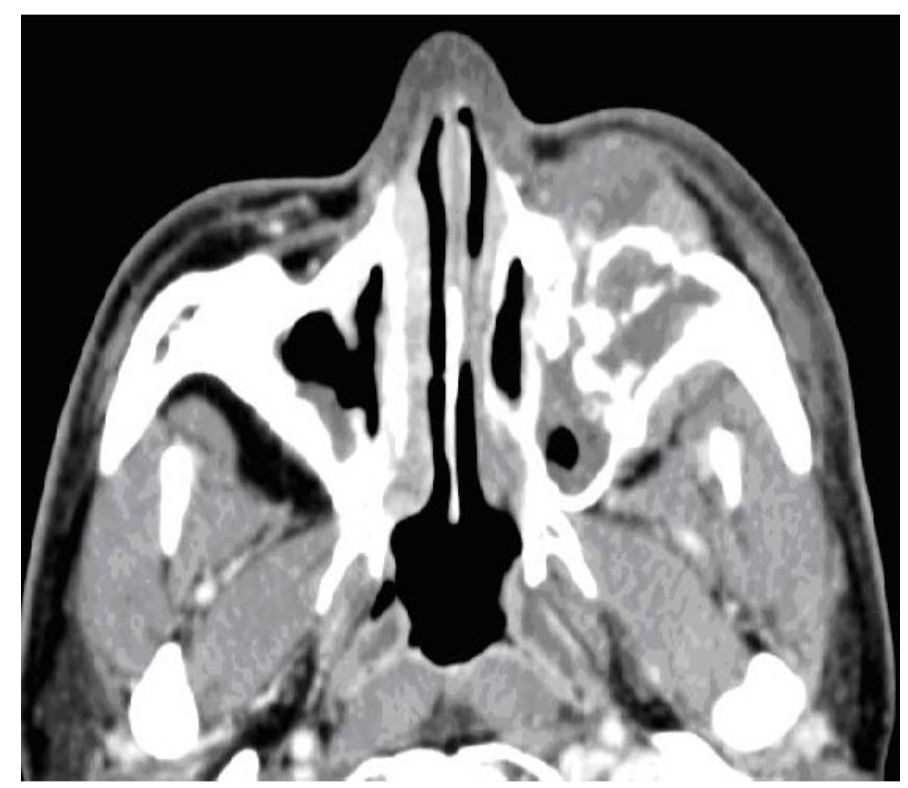

Figure 1. CT scan showing a soft tissue mass occupying the left maxillary sinus with destruction of the maxillary bony wall. 


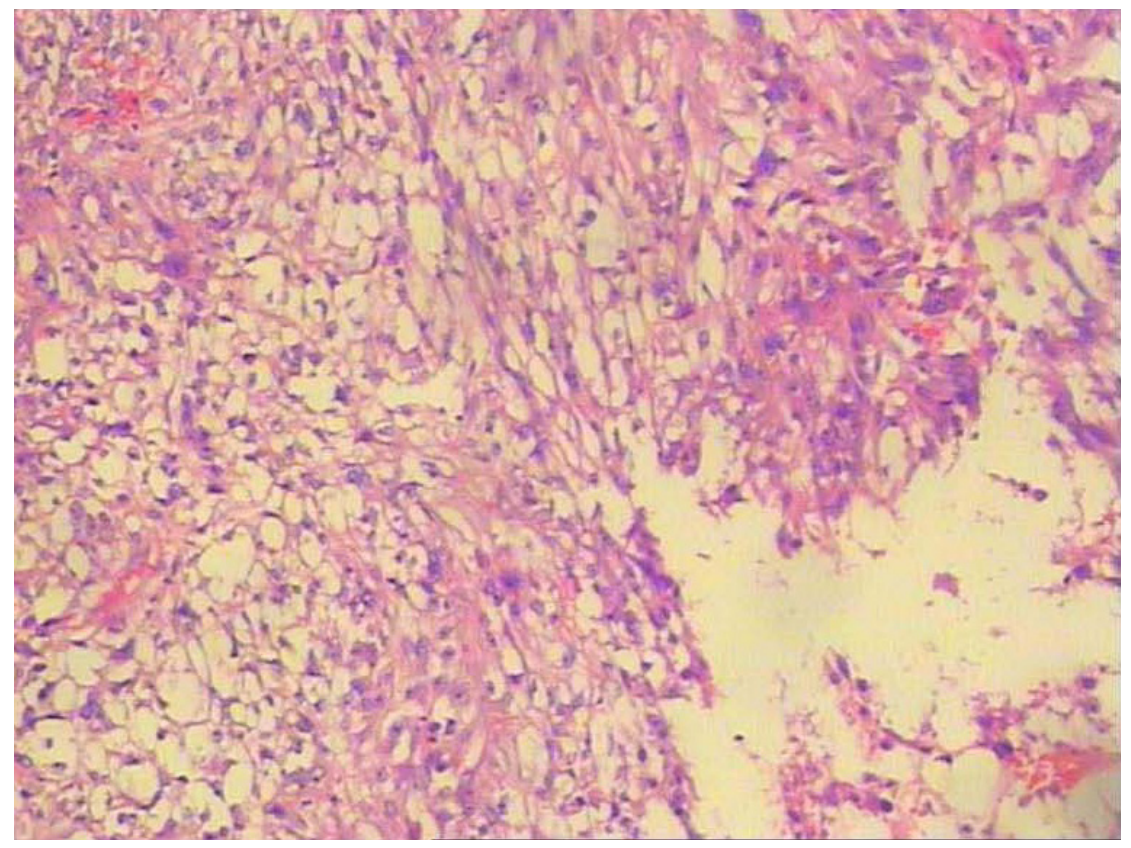

Figure 2. Histologic findings showing metastatic renal cell carcinoma, with characteristically clear cytoplasm (HE 400X).

To confirm the presence of a renal malignancy, further studies were performed. Urinalysis revealed microscopic hematuria. Chest/abdomen/pelvis CT scans showed a $3 \times 4 \times 3 \mathrm{~cm}$ mass on the inferior pole of the left kidney, with extension to the left urinary tract.

The patient underwent radical nephrectomy of the left kidney. The pathology result was consistent with renal cell carcinoma. Since the lesion was invading the bone, external beam radiotherapy ( 30 Gy) was administered in 15 fractions, even though these lesions are not noted for their radiosensitivity. The patient died of disease two years after the primary diagnosis of hypernephroma.

\section{DISCUSSION}

When a neoplastic condition is detected in the paranasal sinuses, a primary paranasal tumor is first suspected, but metastatic tumors from other primary sites should also be considered. Of all primary tumors that arise below the level of the clavicle, renal cell carcinoma is overall the third most common neoplasm to metastasize to the head and neck region, after lung and breast carcinomas, and most of these metastases occur in the thyroid gland. However, renal cell carcinoma is the most frequent infraclavicular tumor to metastasize to the nasal cavity and paranasal sinuses. Therefore, even though it is rare for renal cancer to metastasize to the nasal or paranasal sinuses, renal cancer must be first suspected when investigating metastatic tumors in this region.

There are two routes for renal cancer to metastasize to the nasal and paranasal sinuses. One is the caval route in which tumor cells travel through the inferior vena cava, the right heart, the lungs, the left heart and the maxillary artery to reach the nasal and paranasal sinuses (Nahum and Bailey, 1963). The other is the vertebral plexus route, in which tumor cells do not 
flow into the inferior vena cava, but travel through the venous plexus, the intracranial venous plexus and the cavernous venous plexus to reach the nasal and paranasal sinuses. Emboli can enter the cranial vault through a combination of anterograde and retrograde flow in the intracranial vascular sinuses, arriving at the internal jugular vein, where further unusual flow patterns would allow the emboli to seed structures and develop metastasis in the paranasal sinuses (Gottlieb and Roland Jr, 1998). The vertebral venous plexus was believed to have been involved in our patient, since lung or liver metastasis was not seen.

Renal clear cell carcinoma may metastasize when the diameter of the primary tumor exceeds $3 \mathrm{~cm}$. Because of the slow growth at the initial stage in about $60 \%$ of the cases, the primary lesion receives little attention until symptoms occur because of metastasis (Lokich and Harrison, 1975).

When cancer is diagnosed in a paranasal sinus, a thorough search for a distant primary tumor must be made to rule out metastatic disease. Renal cell carcinomas are known to metastasize early, and symptoms due to the metastatic lesion may be the initial and only manifestation. Twenty-five percent of patients with renal cell carcinoma have distant metastatic lesions at the time of initial assessment.

The original classic triad of flank pain, hematuria, and palpable mass occurs in only about $10 \%$ of patients, and palpable mass occurs in only about $10 \%$ of those patients. However, intermittent microscopic hematuria occurs in 90\% of patients (Skinner et al., 1971). Since symptoms of metastatic tumors often precede those of a primary tumor in renal cancer, a mass in the paranasal sinuses must be diagnosed with this point in mind, even in the absence of a past history of renal cancer.

Even though many metastatic tumors originating from renal cancer develop in multiples, most metastatic tumors in the nasal or paranasal sinuses are single and treated surgically (Matsumoto and Yanagihara, 1982). Patients with a single resectable metastatic lesion should be treated aggressively, since they have an excellent chance for extended survival before further progression of this disease. Non-surgical treatment modalities such as chemotherapy, immunotherapy, radiotherapy, and chemotherapy have significantly failed to improve overall survival, and where possible, surgery is the mainstay of treatment and offers the best hope for survival (Dineen et al., 1988). However, The U.S. Food and Drug Administration approved three kinase inhibitors, sorafenib, sunitinib, and temsirolimus, for treating patients with metastatic renal cell carcinoma (Escudier et al., 2007; Motzer et al., 2007; Hudes et al., 2007).

In conclusion, the case described here is unusual because the primary tumor had not declared its presence and also because of its unusual site. Early diagnosis and surgical removal of both primary and metastatic tumors are important to prolong the patient's life. When a patient presents with a mass in the maxillary region, the possibility of metastatic renal cell carcinoma should be considered.

\section{ACKNOWLEDGMENTS}

We thank Prof. Shi-Cheng Xu and Hang-Cheng Zhou for collecting data. No funding/ sponsorship was received in relation to this study.

\section{REFERENCES}

Dineen MK, Pastore RD, Emrich LJ and Huben RP (1988). Results of surgical treatment of renal cell carcinoma with solitary metastasis. J. Urol. 140: 277-279.

Escudier B, Eisen T, Stadler WM, Szczylik C, et al. (2007). Sorafenib in advanced clear-cell renal-cell carcinoma. N. Engl. 
J. Med. 356: 125-134.

Gottlieb MD and Roland JT Jr. (1998). Paradoxical spread of renal cell carcinoma to the head and neck. Laryngoscope 108: 1301-1305.

Hefer T, Joachims HZ and Golz A (1994). Metastatic renal cell carcinoma to the nose. Eur. Arch. Otorhinolaryngol. 251: 127-129.

Hudes G, Carducci M, Tomczak P, Dutcher J, et al. (2007). Temsirolimus, interferon alfa, or both for advanced renal-cell carcinoma. N. Engl. J. Med. 356: 2271-2281.

Lokich JJ and Harrison JH (1975). Renal cell carcinoma: natural history and chemotherapeutic experience. J. Urol. 114: 371-374.

Matsumoto Y and Yanagihara N (1982). Renal clear cell carcinoma metastatic to the nose and paranasal sinuses. Laryngoscope 92: 1190-1193.

Miyamoto R and Helmus C (1973). Hypernephroma metastatic to the head and neck. Laryngoscope 83: 898-905.

Motzer RJ, Hutson TE, Tomczak P, Michaelson MD, et al. (2007). Sunitinib versus interferon alfa in metastatic renal-cell carcinoma. N. Engl. J. Med. 356: 115-124.

Nahum AM and Bailey BJ (1963). Malignant tumors metastatic to the paranasal sinuses: case report and review of the literature. Laryngoscope 73: 942-953.

Skinner DG, Colvin RB, Vermillion CD, Pfister RC, et al. (1971). Diagnosis and management of renal cell carcinoma. A clinical and pathologic study of 309 cases. Cancer 28: 1165-1177. 